A N N A LES

UNIVERSITATIS MARIAE CURIE-SKŁODOWSKA

LUBLIN - POLONIA

VOL. LXII, 2008

SECTIO A

$75-80$

JAN KUREK and WŁODZIMIERZ M. MIKULSKI

\title{
The natural operators lifting vector fields to the bundle of affinors
}

\begin{abstract}
All natural operators $T \rightsquigarrow T\left(T \otimes T^{*}\right)$ lifting vector fields $X$ from $n$-dimensional manifolds $M$ to vector fields $B(X)$ on the bundle of affinors $T M \otimes T^{*} M$ are described.
\end{abstract}

1. Introduction. In [3], the second author studied the problem how a 1form $\omega$ on an $n$-manifold $M$ induces a 1-form $B(\omega)$ on $T M \otimes T^{*} M$. This problem was reflected in natural operators $B: T^{*} \rightsquigarrow T^{*}\left(T \otimes T^{*}\right)$ over $n$ manifolds. It is proved that the set of natural operators $T^{*} \rightsquigarrow T^{*}\left(T \otimes T^{*}\right)$ over $n$-manifolds is a free $C^{\infty}\left(\mathbf{R}^{n}\right)$-module of dimension $2 n$, and there is presented a basis of this module.

In this note we study a similar problem how a vector field $X$ on an $n$ manifold $M$ induces a vector field $B(X)$ on $T M \otimes T^{*} M$. This problem is reflected in natural operators $T \rightsquigarrow T\left(T \otimes T^{*}\right)$ over $n$-manifolds. We prove that the set of natural operators $T \rightsquigarrow T\left(T \otimes T^{*}\right)$ over $n$-manifolds is a free $C^{\infty}\left(\mathbf{R}^{n}\right)$-module of dimension $n+1$. We construct a basis of this module.

We recall that a natural operator $B: T \rightsquigarrow T\left(T \otimes T^{*}\right)$ over $n$-manifolds is an $\mathcal{M} f_{n}$-invariant family of regular operators

$$
B: \mathcal{X}(M) \rightarrow \mathcal{X}\left(T M \otimes T^{*} M\right)
$$

2000 Mathematics Subject Classification. 58A20, 53A55.

Key words and phrases. Natural bundles, natural operators. 
for all $n$-manifolds $M$. The invariance means that if vector fields $X_{1}$ on $M$ and $X_{2}$ on $N$ are $\varphi$-related for some local diffeomorphism $\varphi: M \rightarrow N$ between $n$-manifolds then the vector fields $B\left(X_{1}\right)$ and $B\left(X_{2}\right)$ are $T \varphi \otimes T^{*} \varphi$ related. The regularity means that $B$ transforms smoothly parametrized families of vector fields into smoothly parametrized families of vector fields.

From now on $x^{1}, \ldots, x^{n}$ are the usual coordinates on $\mathbf{R}^{n}$ and $\partial_{i}=\frac{\partial}{\partial x^{i}}$ for $i=1, \ldots, n$ are the canonical vector fields on $\mathbf{R}^{n}$.

All manifolds and maps are assumed to be of class $C^{\infty}$.

\section{Examples of natural operators $T \rightsquigarrow T\left(T \otimes T^{*}\right)$.}

Example 2.1. Let $X$ be a vector field on an $n$-manifold $M$. Let $\mathcal{T} \otimes \mathcal{T}^{*} X$ be the flow lifting of $X$ to $T M \otimes T^{*} M$. More precisely, if $\varphi_{t}$ is the flow of $X$, then $T \varphi_{t} \otimes T^{*} \varphi_{t}$ is the flow of $\mathcal{T} \otimes \mathcal{T}^{*} X$. The correspondence $\mathcal{T} \otimes \mathcal{T}^{*}$ : $T \rightsquigarrow T\left(T \otimes T^{*}\right)$ given by $X \rightarrow \mathcal{T} \otimes \mathcal{T}^{*} X$ is a natural operator (called the flow operator) in question.

Example 2.2. For $k=0, \ldots, n-1$ we have the canonical vector field $L^{k}$ on $T M \otimes T^{*} M$ such that

$$
L^{k}(A)=\left.\frac{d}{d t}\right|_{0}\left(A+t A^{k}\right), \quad A \in \operatorname{End}\left(T_{x} M\right)=T_{x} M \otimes T_{x}^{*} M, \quad x \in M,
$$

where $A^{k}$ is the $k$-th power of $A\left(A^{0}=i d\right)$. The vector field $L^{k}$ will be called the $k$-th Liouville vector field on $T M \otimes T^{*} M\left(L^{1}\right.$ is the classical Liouville vector field on $\left.T M \otimes T^{*} M\right)$. The correspondence $L^{k}: T \rightsquigarrow T\left(T \otimes T^{*}\right)$ is a natural operator in question.

3. The $C^{\infty}\left(\mathrm{R}^{n}\right)$-module of natural operators $T \rightsquigarrow T\left(T \otimes T^{*}\right)$ over $\boldsymbol{n}$-manifolds. If $L: V \rightarrow V$ is an endomorphism of an $n$-dimensional vector space $V$ then $a_{1}(L), \ldots, a_{n}(L)$ denote the coefficient of the characteristic polynomial

$$
W_{L}(\lambda)=\operatorname{det}\left(\lambda i d_{V}-L\right)=\lambda^{n}+a_{1}(L) \lambda^{n-1}+\cdots+a_{n-1}(L) \lambda+a_{n}(L) .
$$

Thus for every $n$-manifold $M$ we have maps $a_{1}, \ldots, a_{n}: T M \otimes T^{*} M \rightarrow \mathbf{R}$ $\left(\right.$ as $\left.T_{x} M \otimes T_{x}^{*} M=\operatorname{End}\left(T_{x} M\right)\right)$.

The vector space of all natural operators $B: T \rightsquigarrow T\left(T \otimes T^{*}\right)$ over $n$ manifolds is additionally a module over the algebra $C^{\infty}\left(\mathbf{R}^{n}\right)$ of smooth maps $\mathbf{R}^{n} \rightarrow \mathbf{R}$. Actually given a smooth map $f: \mathbf{R}^{n} \rightarrow \mathbf{R}$ and a natural operator $B: T \rightsquigarrow T\left(T \otimes T^{*}\right)$ we have natural operator $f B: T \rightsquigarrow T\left(T \otimes T^{*}\right)$ given by

$$
(f B)(X)=f\left(a_{1}, \ldots, a_{n}\right) B(X)
$$

for any vector field $X$ on an $n$-manifold $M$. 
4. The main result. The main result of this short note is the following classification theorem.

Theorem 1. The flow operator $\mathcal{T} \otimes \mathcal{T}^{*}$ together with the $k$-th Liouville operators $L^{k}$ for $k=0, \ldots, n-1$ form a basis of the $C^{\infty}\left(\mathbf{R}^{n}\right)$-module of natural operators $T \rightsquigarrow T\left(T \otimes T^{*}\right)$ over $n$-manifolds.

The proof of Theorem 1 will occupy the rest of this note.

5. The result of J. Dębecki. The vector space $\operatorname{End}\left(\mathbf{R}^{n}\right)$ of all endomorphisms of $\mathbf{R}^{n}$ is a $G L(n)$-space because of the usual (adjoint) action of $G L(n)$ on $\operatorname{End}\left(\mathbf{R}^{n}\right)$.

We have the following result of J. Dębecki.

Proposition 1 ([1]). Any $G L(n)$-equivariant map

$$
C: \operatorname{End}\left(\mathbf{R}^{n}\right) \rightarrow \operatorname{End}\left(\mathbf{R}^{n}\right)
$$

is of the form

$$
C(A)=\sum_{k=0}^{n-1} f_{k}\left(a_{1}(A), \ldots, a_{n}(A)\right) A^{k}
$$

for some uniquely determined maps $f_{k}: \mathbf{R}^{n} \rightarrow \mathbf{R}$.

6. The vertical type natural operators $B: T \rightsquigarrow T\left(T \otimes T^{*}\right)$ over $\boldsymbol{n}$-manifolds. A natural operator $B: T \rightsquigarrow T\left(T \otimes T^{*}\right)$ is of vertical type if $B(X)$ is a vertical vector field for any vector field $X$ on a $n$-manifold.

Using Proposition 1 we prove the following fact.

Proposition 2. The $C^{\infty}\left(\mathbf{R}^{n}\right)$-submodule of vertical type natural operators $B: T \rightsquigarrow T\left(T \otimes T^{*}\right)$ over $n$-manifolds is free and $n$-dimensional. The $k$-th Liouville operators $L^{k}$ for $k=0, \ldots, n-1$ form a basis of this module.

Proof. Let $B: T \rightsquigarrow T\left(T \otimes T^{*}\right)$ be a vertical type natural operator over $n$-manifolds. Because of the naturality and the Frobenius theorem this operator is uniquely determined by the restriction of vertical vector field $B\left(\partial_{1}\right)$ to the fiber $\operatorname{End}\left(T_{0} \mathbf{R}^{n}\right)=T_{0} \mathbf{R}^{n} \times T_{0}^{*} \mathbf{R}^{n}$.

Using the naturality of $B$ with respect to the homotheties $t i d_{\mathbf{R}^{n}}$ for $t \neq 0$ we see that

$$
B\left(\partial_{1}\right)_{\mid \operatorname{End}\left(T_{0} \mathbf{R}^{n}\right)}=B\left(t \partial_{1}\right)_{\mid \operatorname{End}\left(T_{0} \mathbf{R}^{n}\right)}
$$

for $t \neq 0$. Putting $t \rightarrow 0$ we see that

$$
B\left(\partial_{1}\right)_{\mid \operatorname{End}\left(T_{0} \mathbf{R}^{n}\right)}=B(0)_{\mid \operatorname{End}\left(T_{0} \mathbf{R}^{n}\right)} .
$$

Because of the naturality of $B(0)$ with respect to linear automorphisms of $\mathbf{R}^{n}$ we have a $G L(n)$-equivariant map

$$
C: \operatorname{End}\left(T_{0} \mathbf{R}^{n}\right) \rightarrow \operatorname{End}\left(T_{0} \mathbf{R}^{n}\right)
$$


given by

for $A \in \operatorname{End}\left(T_{0} \mathbf{R}^{n}\right)$.

$$
B(0)(A)=\left.\frac{d}{d t}\right|_{0}(A+t C(A))
$$

By Proposition 1 we have that

$$
C(A)=\sum_{k=0}^{n-1} f_{k}\left(a_{1}(A), \ldots, a_{n}(A)\right) A^{k}
$$

for some uniquely determined maps $f_{k}: \mathbf{R}^{n} \rightarrow \mathbf{R}$. Then

$$
B\left(\partial_{1}\right)(A)=\sum_{k=0}^{n-1} f_{k}\left(a_{1}(A), \ldots, a_{n}(A)\right) L^{k}(A)
$$

for all $A \in \operatorname{End}\left(T_{0} \mathbf{R}^{n}\right)$. That is why $B=\sum_{k=0}^{n-1} f_{k} L^{k}$, as well.

7. Proof of Theorem 1. It is clear that Theorem 1 will be proved after proving the following fact.

Proposition 3. Let $B: T \rightsquigarrow T\left(T \otimes T^{*}\right)$ be a natural operator over $n$ manifolds. Then there exists a unique map $f: \mathbf{R}^{n} \rightarrow \mathbf{R}$ such that $B-f \mathcal{T} \otimes$ $\mathcal{T}^{*}$ is a vertical type operator.

Let $\pi: T \mathbf{R}^{n} \otimes T^{*} \mathbf{R}^{n} \rightarrow \mathbf{R}^{n}$ be the bundle projection.

Lemma 1. There exist unique maps $f_{k} \in C^{\infty}\left(\mathbf{R}^{n}\right)$ such that

$$
T \pi\left(B\left(w^{o}\right)(A)\right)=\sum_{k=0}^{n-1} f_{k}\left(a_{1}(A), \ldots, a_{n}(A)\right) A^{k}(w)
$$

for $A \in \operatorname{End}\left(T_{0} \mathbf{R}^{n}\right)=T_{0} \mathbf{R}^{n} \otimes T_{0}^{*} \mathbf{R}^{n}$ and $w \in T_{0} \mathbf{R}^{n}$, where $w^{o}$ is the "constant" vector field on $\mathbf{R}^{n}$ with $w_{0}^{o}=w$.

Proof. By the invariance of $B$ with respect to the homotheties $t i d_{\mathbf{R}^{n}}$ for $t \neq 0$ we have the homogeneity condition

$$
T \pi\left(B\left((t w)^{o}\right)(A)=t T \pi\left(B\left(w^{o}\right)\right)(A) .\right.
$$

Then by the homogeneous function theorem, [2], $T \pi\left(B\left(w^{o}\right)(A)\right.$ depends linearly on $w$.

So, we can define a map $C: \operatorname{End}\left(T_{0} \mathbf{R}^{n}\right) \rightarrow \operatorname{End}\left(T_{0} \mathbf{R}^{n}\right)$ by

$$
C(A)(w)=T \pi\left(B\left(w^{o}\right)(A)\right)
$$

for all $A \in \operatorname{End}\left(T_{0} \mathbf{R}^{n}\right)$ and $w \in T_{0} \mathbf{R}^{n}$.

Because of the naturality of $B$ with respect to linear automorphisms of $\mathbf{R}^{n}, C$ is $G L(n)$-equivariant. Then applying Proposition 1 we end the proof.

Lemma 2. Let $B: T \rightsquigarrow T\left(T \otimes T^{*}\right)$ be as in Lemma 1. Let $f_{0}, \ldots, f_{n-1}$ be the maps from Lemma 1 . Then $f_{j}=0$ for $j=1, \ldots, n-1$. 
Proof. Consider $j=1, \ldots, n-1$. Let $b=\left(b_{1}, \ldots, b_{n}\right) \in \mathbf{R}^{n}$. Let $A \in$ $\operatorname{End}\left(T_{0} \mathbf{R}^{n}\right)$ be such that $A\left(\partial_{i}(0)\right)=\partial_{i+1}(0)$ for $i=1, \ldots, n-1$ and $A\left(\partial_{n}(0)\right)=-b_{n} \partial_{1}(0)-\ldots-b_{1} \partial_{n}(0)$. Then $a_{i}(A)=b_{i}$ for $i=1, \ldots, n$.

Let $\varphi_{t}=\left(x^{1}, \ldots, x^{j+1}+t x^{j+1}+\ldots, \ldots, x^{n}\right)$ be the flow of $\partial_{j+1}+x^{j+1} \partial_{j+1}$ near $0 \in \mathbf{R}^{n}$.

Since $T_{0} \varphi_{1} \circ A \circ T_{0} \varphi_{1}^{-1} \neq A$ (as the left hand side evaluated at $\partial_{j}(0)$ is equal to $2 \partial_{j+1}(0)$ and the right hand side evaluated in the same vector $\partial_{j}(0)$ is equal to $\left.\partial_{j+1}(0)\right)$, we have

$$
\mathcal{T} \otimes \mathcal{T}^{*}\left(x^{j+1} \partial_{j+1}\right)(A) \neq 0 .
$$

Using the Zajtz theorem [4], since $\left(\partial_{j+1}+x^{j+1} \partial_{j+1}\right)(0)=\partial_{j+1}(0) \neq 0$, we find a diffeomorphism $\eta: \mathbf{R} \rightarrow \mathbf{R}$ such that

$$
j_{0}^{1} \psi=i d
$$

and

$$
\psi_{*} \partial_{j+1}=\partial_{j+1}+x^{j+1} \partial_{j+1}
$$

near $0 \in \mathbf{R}^{n}$, where $\psi\left(x^{1}, \ldots, x^{n}\right)=\left(x^{1}, \ldots, x^{j}, \eta\left(x^{j+1}\right), \ldots, x^{n}\right)$.

Clearly $\psi$ preserves $\partial_{1}$. Because of $(2), \psi$ preserves $A$. Then $\psi$ preserves $B\left(\partial_{1}\right)(A)$.

Because of (2), $\psi$ preserves any vertical vector tangent to $T \mathbf{R}^{n} \otimes T^{*} \mathbf{R}^{n}$ at $A$. Moreover, $\psi$ preserves all $\partial_{l}$ for $l=1, \ldots, n$ with $l \neq j+1$. By $(3), \psi$ sends $\mathcal{T} \otimes \mathcal{T}^{*}\left(\partial_{j+1}\right)(A)$ into $\mathcal{T} \otimes \mathcal{T}^{*}\left(\partial_{j+1}+x^{j+1} \partial_{j+1}\right)(A)$. Then $\psi$ sends

$B\left(\partial_{1}\right)(A)=\sum_{k=0}^{n-1} f_{k}\left(a_{1}(A), \ldots, a_{n}(A)\right) \mathcal{T} \otimes \mathcal{T}^{*}\left(\partial_{k+1}\right)(A)+$ some vertical vector

into $B\left(\partial_{1}\right)(A)+f_{j}(b) \mathcal{T} \otimes \mathcal{T}^{*}\left(x^{j+1} \partial_{j+1}\right)(A)$.

Then because of $(1)$, we have $f_{j}(b)=0$, as well.

Proof of Proposition 3. Because of Lemmas 1 and 2 we have

$$
B\left(\partial_{1}\right)(A)=f_{0}\left(a_{1}(A), \ldots, a_{n}(A)\right) \mathcal{T} \otimes \mathcal{T}^{*}\left(\partial_{1}\right)(A)+\text { some vertical vector }
$$

for any $A \in \operatorname{End}\left(T_{0} \mathbf{R}^{n}\right)$. Since $B$ is determined by $B\left(\partial_{1}\right)$ over 0 , the proof of Proposition 3 is complete.

\section{REFERENCES}

[1] Dębecki, J., Natural transformations of affinors into functions and affinors, Suppl. Rend. Circolo Mat. Palermo 30(II) (1993), 101-112.

[2] Kolář, I., Michor, P. W. and Slovák, J., Natural Operations in Differential Geometry, Springer-Verlag, Berlin, 1993.

[3] Mikulski, W. M., Liftings of 1-forms to the bundle of affinors, Ann. Univ. Mariae Curie-Skłodowska Sect. A 65 (2001), 109-113.

[4] Zajtz, A., On the order of natural differential operators and liftings, Ann. Polon. Math. 49 (1988), 169-175. 
Jan Kurek

Institute of Mathematics

Maria Curie-Skłodowska University

pl. Marii Curie-Skłodowskiej 1

20-031 Lublin, Poland

e-mail: kurek@hektor.umcs.lublin.pl

Received January 4, 2008
Włodzimierz M. Mikulski

Institute of Mathematics

Jagiellonian University

ul. Łojasiewicza 6

30-348 Kraków, Poland

e-mail: mikulski@im.uj.edu.pl 